\title{
Control of electron spin and orbital resonances in quantum dots through spin-orbit interactions
}

\author{
Peter Stano ${ }^{1,2}$ and Jaroslav Fabian ${ }^{1}$ \\ ${ }^{1}$ Institute for Theoretical Physics, University of Regensburg, 93040 Regensburg, Germany \\ ${ }^{2}$ Research Center for Quantum Information, Slovak Academy of Sciences, 84511 Bratislava, Slovakia
}

(Received 10 November 2006; revised manuscript received 9 August 2007; published 10 January 2008)

\begin{abstract}
The influence of a resonant oscillating electromagnetic field on a single electron in coupled lateral quantum dots in the presence of phonon-induced relaxation and decoherence is investigated. Using symmetry arguments, it is shown that the spin and orbital resonances can be efficiently controlled by spin-orbit interactions. The control is possible due to the strong sensitivity of the Rabi frequency to the dot configuration (the orientation of the dot and the applied static magnetic field); the sensitivity is a result of the anisotropy of the spin-orbit interactions. The so-called easy passage configuration is shown to be particularly suitable for a magnetic manipulation of spin qubits, ensuring long spin relaxation times and protecting the spin qubits from electric field disturbances accompanying on-chip manipulations.
\end{abstract}

DOI: 10.1103/PhysRevB.77.045310

PACS number(s): 73.63.Kv, 76.30.-v, 71.70.Ej

\section{INTRODUCTION}

The spin properties of few-electron quantum dots have recently been extensively studied, in the hope that a localized spin can serve as a qubit, a central building block of a quantum computer. ${ }^{1-4}$ Spin, compared to orbital degrees of freedom, was anticipated to have a much longer coherence time. ${ }^{5}$ Fast experimental progress during the last few years supported this assumption-long electron spin relaxation ${ }^{6-9}$ and dephasing times ${ }^{10-12}$ have been measured in quantum dots.

If a quantum dot electron spin is to form a qubit, DiVincenzo's criteria have to be fulfilled: ${ }^{13}$ (i) The existence of a qubit - the two states of an electron spin naturally encode the information bit. (ii) The initialization of the qubit-in the presence of a magnetic field, at low enough temperatures, the system relaxes into the spin-polarized ground state spontaneously. (iii) Readout-this can be done by a spin-to-charge conversion scheme. ${ }^{14,15}$ (iv) Coherent manipulation-a first important step toward efficient individual spin manipulation has been the recent demonstration of magnetically driven Rabi oscillations. ${ }^{16}$ The above criteria for an electron spin in a quantum dot to work as a qubit have been met at the proofof-the-principle level. The current effort is aimed at the integration of the requirements, with the final goal of a (v) scalable qubit design.

In a scalable architecture, the quantum dots in an array should be addressed individually. This places stringent constraints on the spatial extent of the manipulating electromagnetic fields-one speaks of fields generated on chip. If the spin is manipulated by a magnetic field which is produced locally (say, by an oscillating current in a wire nearby the dot), the electron inevitably feels an accompanying oscillating electric field. This electric field appears due to imperfect screening of the dot from the circuitry-the field produced directly by the changing magnetic field, $\nabla \times \mathbf{E}=-\partial_{t} \mathbf{B}$, is negligible. ${ }^{17}$ The electric field strongly disturbs the orbital part of the electron wave function and, if spin-orbit coupling is present, also couples to the spin, imposing limits on the strength of the applied magnetic field (in Ref. 16, this limit was $1.9 \mathrm{mT}$ ). As a result, the speed of the operation, given by the maximal achievable Rabi frequency, is limited as well.
Fortunately, the accompanying electric fields can be helpful for the qubit control. Through the spin-orbit interaction, the field induces similar spin oscillations ${ }^{17-19}$ as result from magnetic fields. From a practical point of view, the electric fields here are even preferable, since one can control them better than magnetic fields; the possibility of electrically induced spin oscillations is currently being pursued experimentally. However, there is a drawback, since the electric Rabi frequency is expected to depend on dot parameters, primarily through materials dependent spin-orbit coupling. The Rabi frequencies due to magnetic fields depend on the field strength alone.

How effective are, in comparison, oscillating electric and magnetic fields in inducing Rabi spin oscillations? This is a question of primary relevance for undergoing experiments. We need to know how large fields are required to induce Rabi oscillation of a given frequency, as well as how the Rabi frequency depends, for a given field strength, on the parameters of the dot and especially, how stable the oscillations are against the fluctuations of these parameters. Our paper aims to answer the above questions. In particular, we quantify the dipolar electric and magnetic couplings in the spin resonance of a single electron confined in a quantum dot. We consider the textbook resonance scheme in which the ground state of an electron is Zeeman split by an applied static magnetic field. The oscillations between the two split states are induced by oscillating magnetic and electric fields, with the resonance achieved if the field frequency equals to the Zeeman energy.

It has already been proposed theoretically that due to the presence of the spin-orbit interactions in a single quantum dot, the electric field can indeed be effective in inducing spin resonance. ${ }^{17}$ For a typical lateral single GaAs quantum dot, in a static magnetic field of $1 \mathrm{~T}$, an oscillating electric field of $10^{3} \mathrm{~V} / \mathrm{m}$ is as effective as an oscillating magnetic field of $1 \mathrm{mT}$. Here, we consider a more general case of the experimentally relevant double dot setup. Our main result is that the anisotropy of the spin-orbit interactions, which reflects the anisotropy of the underlying GaAs heterostructure, allows for an effective control over the electric field efficiency in spin manipulations. Our findings provide guidelines for dot configurations for two possible strategies: If a local elec- 
tric field is chosen for the spin manipulation, we show how its efficiency can be maximized. If a magnetic field is chosen, the coupling due to the accompanying electric field is unwanted and we show that the electric effects can be suppressed by lowering the magnitude of the static magnetic field and/or by orientating the magnetic field along certain symmetry directions. Particularly, in an easy passage configuration, ${ }^{20}$ the otherwise most effective electric field component can be completely blocked from disturbing the spin.

While the spin resonance study is our main goal, we also investigate the electrically and magnetically induced orbital resonances, in which the resonant states are the two lowest orbital states of the same spin. A qubit represented by these two states is called a charge qubit. Our study is motivated by an observation, that in the presence of the spin-orbit interactions, an analog to electrically induced spin resonance should exist; the magnetic field should be able to induce oscillation between spin alike states. We show that this is indeed true. However, for realistic values of the materials parameters, the magnetic field is, expectedly, much less effective than the electric field.

We use realistic parameters for electrically defined single and coupled lateral dots defined in a [001] grown GaAs heterostructure. We treat the problem numerically by an exact diagonalization of the full single-electron Hamiltonian. We support our numerical results with analytical arguments based on an effective spin-orbit Hamiltonian and the degenerate perturbation theory. Our model incorporates the electron relaxation and decoherence rates caused by acoustic phonons in a realistic way; the rates we use have been found to be in a very good agreement with the experimental data for magnetic fields above $1 \mathrm{~T}$ both in single $\mathrm{e}^{7,20}$ and double dots. ${ }^{8}$

The paper is organized as follows. In Sec. II, we describe the model of the electron in the dissipative phonon environment under oscillating electric and magnetic fields. In Sec. III, we derive an effective spin-orbit Hamiltonian which allows a symmetry analysis of the problem. With this Hamiltonian, we evaluate the matrix elements of the oscillating magnetic and electric fields for the case of spin (Sec. IV) and orbital (Sec. V) resonances. Finally, in Sec. VI, we describe the system in the steady state, where we show how to obtain the Rabi frequency and decoherence rates from a steady state measurement.

\section{MODEL}

Consider a single electron in a double quantum $\operatorname{dot}^{21}$ formed in a two dimensional electron gas in a (001) plane of a GaAs/AlGaAs heterostructure. The effective Hamiltonian is

$$
H=H_{0}+H_{B R}+H_{D}+H_{D 3},
$$

where

$$
H_{0}=T+V_{C}+H_{Z} \text {. }
$$

The kinetic energy is $T=\hbar \mathbf{k}^{2} / 2 m$ with the effective electron mass $m$ and kinetic momentum $\hbar \mathbf{k}=-i \hbar \nabla$. The double quan-

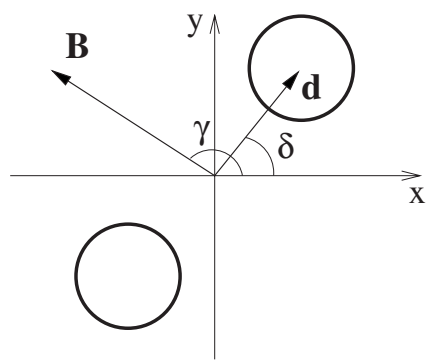

FIG. 1. The orientation of the potential dot minima (denoted as the two circles) with respect to the crystallographic axes $(x=[100]$ and $y=[010])$ is defined by the angle $\delta$. The orientation of the inplane magnetic field is given by the angle $\gamma$.

tum dot is described here by the confinement

$$
V_{C}(\mathbf{r})=\frac{1}{2} m \omega_{0}^{2} \min \left\{(\mathbf{r}-\mathbf{d})^{2},(\mathbf{r}+\mathbf{d})^{2}\right\},
$$

representing two alike potential minima of a parabolic shape, centered at $\pm \mathbf{d}$. The in-plane orientation of the double dot with respect to the crystallographic axes $x$ and $y$ is defined by $\delta$, the angle between $\mathbf{d}$ and $\hat{x}$. A single dot with the confinement_energy $E_{0}=\hbar \omega_{0}$ and the confinement length $l_{0}$ $=m \sqrt{\hbar} / m \omega_{0}$ is defined by the limit $d=0$. Alternatively to giving the interdot distance $d$, the double dot can be characterized by the tunneling energy $\delta E_{t}$ equal to the half of the difference of the energies of the two lowest orbital states. ${ }^{22}$ The electron feels an in-plane magnetic field $\mathbf{B}$ whose orbital effects can be neglected for fields lower than $\sim 10 \mathrm{~T}$. The Zeeman term is $H_{Z}=\mu \sigma \cdot \mathbf{B}$, where $\mu=(g / 2) \mu_{B}$ is the renormalized magneton, $g$ is the conduction band $g$ factor, $\mu_{B}$ is the Bohr magneton, and $\boldsymbol{\sigma}$ are the Pauli matrices. The spin quantization axis is defined by the direction of the magnetic field. The angle between $\mathbf{B}$ and $\hat{x}$ is denoted as $\gamma$. The geometry is summarized in Fig. 1.

The spin-orbit coupling in our confined system is described by three terms. ${ }^{23}$ The Bychkov-Rashba Hamiltonian, ${ }^{24,25}$

$$
H_{B R}=\frac{\hbar^{2}}{2 m l_{B R}}\left(\sigma_{x} k_{y}-\sigma_{y} k_{x}\right),
$$

is present due to the heterostructure asymmetry, while the linear and cubic Dresselhaus Hamiltonians, ${ }^{26,27}$

$$
\begin{aligned}
& H_{D}=\frac{\hbar^{2}}{2 m l_{D}}\left(-\sigma_{x} k_{x}+\sigma_{y} k_{y}\right), \\
& H_{D 3}=\gamma_{c}\left(\sigma_{x} k_{x} k_{y}^{2}-\sigma_{y} k_{y} k_{x}^{2}\right),
\end{aligned}
$$

are due to the lack of the bulk inversion symmetry.

In our numerical calculations, we use bulk GaAs material parameters: $m=0.067 m_{e}, g=-0.44$, and $\gamma_{c}=27.5 \mathrm{eV} \AA^{3}$. For the coupling of the linear spin-orbit terms, we choose $l_{B R}$ $=1.8 \mu \mathrm{m}$ and $l_{D}=1.3 \mu \mathrm{m}$; the values used to fit a recent experiment. ${ }^{20}$ For the confinement length, we take $l_{0}$ $=30 \mathrm{~nm}$, corresponding to the confinement energy $E_{0}$ $=1.2 \mathrm{meV}$. 
We now describe the influence of the phonon environment as well as of the oscillating electric and magnetic fields. The phonon environment leads to the relaxation ${ }^{28-31}$ and decoherence expressed, in the Markov and Born approximations, by the time derivative of the diagonal and off-diagonal elements of the reduced density matrix of the electron $\rho$ (Ref. 32) (upperscript "ph" stands for phonons to discriminate from other contributions to the time derivative which appear later),

$$
\begin{gathered}
\partial_{t}^{\mathrm{ph}} \rho_{i i}=-\sum_{k} 2 \Gamma_{i k} \rho_{i i}+\sum_{k} 2 \Gamma_{k i} \rho_{k k}, \\
\partial_{t}^{\mathrm{ph}} \rho_{i j}=-\sum_{k}\left(\Gamma_{i k}+\Gamma_{j k}\right) \rho_{i j} \equiv-\gamma_{i j} \rho_{i j} .
\end{gathered}
$$

Here, $2 \Gamma_{i j}$ is the relaxation rate from the electron state $i$ to $j$ due to the piezoelectric and deformation potential interactions of the electron with acoustic phonons. There is no additional phonon channel for the decoherence $\gamma_{i j}$ apart from the relaxation since the phonon density of states vanishes for zero phonon energy, $\Gamma_{i i}=0$. We do not consider nonphonon mechanisms of dephasing, which are important at low (subtesla) magnetic fields. To allow for a finite temperature, one can suppose a detailed balance: $\Gamma_{j i}=\tau \Gamma_{i j}$, where $\tau=\exp ($ $\left.-\hbar \omega_{i j} / k_{B} T\right)$. In the calculations below, we consider temperature much lower than the orbital excitation energy. For example, the experiment Ref. 16 was done at temperature of $100 \mathrm{mK}$, corresponding to $\sim 0.01 \mathrm{meV}$, while a typical excitation energy of the used quantum dot was about $1 \mathrm{meV}$. In this limit, a transition into a higher orbital level has a negligible rate.

In addition to phonons, the electron is subject to oscillating electric and magnetic fields, which contribute through the following Hamiltonian:

$$
H^{\mathrm{of}}=[e \mathcal{E} \cdot \mathbf{r}+\mu \mathcal{B} . \boldsymbol{\sigma}] \cos \omega t \equiv \hbar \hat{\Omega} \cos \omega t .
$$

Only the in-plane components of the oscillating electric field are relevant. The oscillating magnetic field is perpendicular to the plane, $\mathcal{B} \propto \hat{z}$, simulating the conditions in the experiment. ${ }^{16}$ In the numerical calculations, we set $E$ $=1000 \mathrm{~V} / \mathrm{m}$ as a realistic guess for the experimental setup ${ }^{33}$ and $B=1 \mathrm{mT}$, a typical value from the experiment. ${ }^{16}$ We suppose that the frequency $\omega$ is close to the energy difference of a given pair of states - resonant states - denoted by indices $a$ and $b$, such that $\omega \approx \omega_{b a}=\left(E_{b}-E_{a}\right) / \hbar>0$. In the rotating wave approximation, ${ }^{32}$ which we adopt, the oscillating field influences only the two resonant states, contributing to the time derivative of the density matrix (superscript "of" stands for the oscillating field),

$$
\begin{gathered}
\partial_{t}^{\mathrm{of}} \rho_{a a}=-\partial_{t}^{\mathrm{of}} \rho_{b b}=\frac{i}{2} \Omega_{b a} \rho_{a b} e^{i \Delta t}-\frac{i}{2} \Omega_{a b} \rho_{b a} e^{-i \Delta t}, \\
\partial_{t}^{\mathrm{of}} \rho_{a b}=-\frac{i}{2}\left(\rho_{b b}-\rho_{a a}\right) \Omega_{a b} e^{-i \Delta t},
\end{gathered}
$$

where $\Delta=\omega_{b a}-\omega$ is the detuning from the resonance.

The time evolution of the density matrix, given by Eqs. (7a), (7b), (9a), and (9b), can be easily solved if one neglects all other states but the two resonant. ${ }^{3,34}$ Such an approxima- tion makes sense if the electron cannot escape from the two state subspace. Roughly speaking, the effective rate out of the subspace must be much smaller than the rates for the transitions restoring the electron in. This, for example, means that the ground state must be one of the resonant states, which is the case here. Another interesting counterexample is optical shelving, ${ }^{35}$ whereby the electron can be trapped in an intermediate dark state-this is a crucial ingredient of an optical spin readout of the nitrogen vacancy centers in diamond. ${ }^{36}$ There are parameter values for our model where the three lowest electron states can realize such a scheme, but we do not discuss it in this paper. We work in the regime in which the two level approximation is justified, as follows also from our numerical results. The validity of the two level approximation also implies that the decoherence rate is given by the relaxation only,

$$
\gamma_{a b}=\gamma_{b a}=\Gamma_{b a}+\Gamma_{a b}=\Gamma_{b a}(1+\tau),
$$

a fact that we will use later.

Suppose now that the electron is in the ground state initially. After the resonant field is turned on, the populations of the two resonant states start to oscillate, meaning that after a certain time, the electron will be in the excited state, then comes back to the ground state, and so on. Since these Rabi oscillations are coherent, they can realize a single qubit rotation, one of the basic building blocks of quantum computation. The time after which the populations switch is proportional to the inverse of the frequency of the Rabi oscillations (Rabi frequency) $\Omega$. A larger Rabi frequency then means a faster single qubit operation. To better assess the suitability for quantum computation, one has to take into account also the decay of the Rabi oscillations due to decoherence. In our model, the magnitude of the oscillations decays exponentially with the rate roughly proportional to the decoherence rate $\gamma_{b a}$. Therefore, to minimize the error in a single qubit operation, it is desirable to maximize the ratio $\Omega / \gamma_{b a}$, which quantifies how many single qubit operations one can do during the decoherence time. We note that from the observed decaying Rabi oscillations in the time domain, ${ }^{10,16}$ both $\Omega$ and $\gamma_{b a}$ can be extracted.

Finally, we note that in the two resonant states approximation, there are three important rates: decoherence $\gamma_{b a}$, detuning $\Delta$, and the field matrix element $\left|\Omega_{b a}\right|$. If the the last one is not dominant, then either $\gamma_{b a}$ is large and the damping is too strong to observe Rabi oscillations or $\Delta$ is large and the magnitude of the oscillations is small ${ }^{3}$ - both cases are not of interest here. We consider the case when the field matrix element is indeed dominant. It holds then that the matrix element equals the Rabi frequency,

$$
\Omega=\left|\Omega_{b a}\right|,
$$

and is therefore of crucial importance. In the next, we analyze in detail how the field matrix element due to electric and magnetic oscillating fields depends on system parameters. To simplify the analysis of the spin-orbit influence, we begin with a derivation of an effective spin-orbit Hamiltonian. 


\section{EFFECTIVE SPIN-ORBIT HAMILTONIAN}

It is useful to remove the linear spin-orbit terms in Eq. (1) by applying a unitary transformation, ${ }^{37}$ leading to a new Hamiltonian,

$$
H^{\prime}=e^{S} H e^{-S}=H_{0}+H_{1},
$$

where

$$
S=\frac{i}{2 l_{B R}}\left(y \sigma_{x}-x \sigma_{y}\right)-\frac{i}{2 l_{D}}\left(x \sigma_{x}-y \sigma_{y}\right)
$$

is a transformation matrix and

$$
H_{1}=H_{D 3}+H_{\text {lin }}^{(2)}+H_{Z}^{(2)}+H_{D 3}^{(2)}
$$

is an effective spin-orbit interaction. In addition to the cubic Dresselhaus term $H_{D 3}, H_{1}$ comprises the following parts:

$$
\begin{gathered}
H_{\text {lin }}^{(2)}=\frac{\hbar^{2}}{4 m}\left(l_{D}^{-2}-l_{B R}^{-2}\right) \sigma_{z}\left(x k_{y}-y k_{x}\right), \\
H_{Z}^{(2)}=-\mu B \sigma_{z}\left(x h_{1}^{x}+y h_{1}^{y}\right), \\
H_{D 3}^{(2)}=-\frac{\gamma_{c}}{2 l_{B R}}\left[4 k_{x} k_{y}-\sigma_{z}\left(\left\{y, k_{y} k_{x}^{2}\right\}-\left\{x, k_{x} k_{y}^{2}\right\}\right)\right]-\frac{\gamma_{c}}{2 l_{D}}\left[\mathbf{k}^{2}\right. \\
\left.+\sigma_{z}\left(\left\{x, k_{y} k_{x}^{2}\right\}-\left\{y, k_{x} k_{y}^{2}\right\}\right)\right] .
\end{gathered}
$$

Higher order terms and a constant factor were omitted in $H_{1}$. The curly brackets denote the anticommutator, while $\mathbf{h}_{1}$ is an effective spin-orbit vector specified below.

For the following discussion, the symmetries of the terms in Eq. (14) are important. First, each term has a definite time reversal symmetry: $H_{Z}^{(2)}$ is antisymmetric, while the other terms are time reversal symmetric. Second, the spatial symmetry of a particular term is defined by a combination of variables $x, y, k_{x}$, and $k_{y}$ it contains. To exploit the spatial symmetry of the confinement [Eq. (3)], we rotate the (originally crystallographic axes) coordinates such that the new $\hat{x}$ lies along $\mathbf{d}$. The coordinates change according to

$$
x \rightarrow x \cos \delta-y \sin \delta, \quad y \rightarrow y \cos \delta+x \sin \delta,
$$

and similarly for $k_{x}$ and $k_{y}$. The rotation leaves Eq. (15) unchanged. In Eq. (16), the effective linear spin-orbit couplings $h_{1}^{x}$ and $h_{1}^{y}$ acquire the following form:

$$
\begin{aligned}
& h_{1}^{x}=l_{B R}^{-1} \cos (\gamma-\delta)-l_{D}^{-1} \sin (\gamma+\delta), \\
& h_{1}^{y}=l_{B R}^{-1} \sin (\gamma-\delta)-l_{D}^{-1} \cos (\gamma+\delta) .
\end{aligned}
$$

It is important that these couplings can be selectively tuned to zero by orienting the static magnetic field $\mathbf{B}$ in a certain direction $(\gamma)$ dependent on the orientation of the double dot $(\delta)$. The result of the rotation in Eq. (17) is not presented here; we will discuss only its most relevant terms.

We can obtain analytical results in reasonable quantitative agreement with the numerics in the lowest order degenerate perturbation theory by exploiting the symmetries of the problem. The orbital eigenfunctions of $H_{0}$ [Eq. (2)] in an in-plane magnetic field form a representation of $C_{2 v}$ symmetry group. ${ }^{22}$ There are four possible symmetry classes which

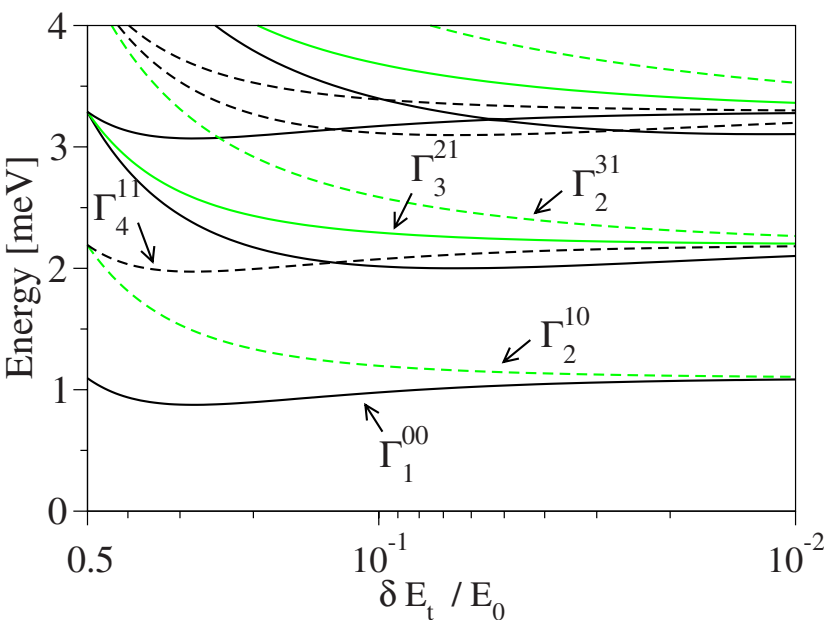

FIG. 2. (Color online) The lowest part of the energy spectrum of Hamiltonian $H_{0}$ [Eq. (2)] in zero magnetic field as a function of the tunneling energy $\left(\delta E_{t}\right)$ in the units of the confinement energy $E_{0}$. Each eigenfunction belongs to one of the four symmetry classes of $C_{2 v}$, which are denoted by different color/type of the line. Spin indices are omitted.

transform upon inversions along (rotated axes) $\hat{x}$ and $\hat{y}$ as 1 , $x, x y$, and $y$, respectively. A relevant part of the double dot spectrum is shown in Fig. 2. Several eigenstates are labeled by $\Gamma$ with indices, where the bottom index denotes the spatial symmetry of the state (four symmetry groups), while the upper indices labels states within the symmetry group-this notation was introduced in Ref. 22. The two lowest orbital states will play the most important role: the ground state $\Gamma_{1}^{00}$ is symmetric both in $x$ and $y$ (often denoted as the bonding molecular orbital), and the first excited orbital state $\Gamma_{2}^{10}$ is antisymmetric in $x$ and symmetric in $y$ (antibonding).

If a magnetic field is applied, each line in Fig. 2 splits into two by the Zeeman term lifting the degeneracy. Assuming a negative $g$ factor (for GaAs quantum dots) and a positive $B$, a spin down state (denoted by $\downarrow$ ) has a higher energy than a spin up state $(\uparrow)$. Another important consequence of a finite Zeeman energy is the anticrossing of states $\Gamma_{1 \downarrow}^{00}$ and $\Gamma_{2 \uparrow}^{10}$, influence of which we take into account using the degenerate perturbation theory. The exact eigenfunctions (denoted by an overline) of the Hamiltonian $H^{\prime}$ can be written as a combination of the solutions of $H_{0}$ (denoted by $\Gamma$ as in Fig. 2): the three lowest states, in the lowest order of the degenerate perturbation theory, are

$$
\begin{gathered}
\bar{\Gamma}_{1 \uparrow}^{00} \approx \Gamma_{1 \uparrow}^{00}+\frac{\left\langle\Gamma_{2 \downarrow}^{10} H_{1} \Gamma_{1 \uparrow}^{00}\right\rangle}{E_{1 \uparrow}^{00}-E_{2 \downarrow}^{10}} \Gamma_{2 \downarrow}^{10}+\cdots, \\
\bar{\Gamma}_{1 \downarrow}^{00} \approx \alpha \Gamma_{1 \downarrow}^{00}+\beta \Gamma_{2 \uparrow}^{10}+\frac{\left\langle\Gamma_{4 \uparrow}^{11} H_{1} \Gamma_{1 \downarrow}^{00}\right\rangle}{E_{1 \downarrow}^{00}-E_{4 \uparrow}^{11}} \Gamma_{4 \uparrow}^{11}+\cdots, \\
\bar{\Gamma}_{2 \uparrow}^{10} \approx \alpha \Gamma_{2 \uparrow}^{10}-\beta^{*} \Gamma_{1 \downarrow}^{00}+\frac{\left\langle\Gamma_{4 \downarrow}^{11} H_{1} \Gamma_{2 \uparrow}^{10}\right\rangle}{E_{2 \uparrow}^{00}-E_{4 \downarrow}^{11}} \Gamma_{4 \downarrow}^{11}+\cdots .
\end{gathered}
$$

The dots denote the rest of an infinite sum through the eigenfunctions of $H_{0}$. The anticrossing is described by the coefficients 


$$
\begin{gathered}
\beta=\arg (c \delta E) \sin [\arctan (|4 c / \delta E|) / 2], \\
\alpha=\sqrt{1-|\beta|^{2}},
\end{gathered}
$$

which depend on the energy difference $\delta E=E_{1 \downarrow}^{00}-E_{2 \uparrow}^{10}$ and the coupling $c=\left\langle\Gamma_{2 \uparrow}^{10} H_{1} \Gamma_{1 \downarrow}^{00}\right\rangle$ between the unperturbed crossing states.

From the above expression for the coupling $c$, it follows that the anticrossing is caused by the part of $H_{1}$ with the spatial symmetry of $x$, which is the symmetry of $\Gamma_{2 \uparrow}^{10}$. After the rotation [Eq. (18)], the only term with the $x$ symmetry in Eqs. (15)-(17) is the first term in $H_{Z}^{(2)}$. Therefore, by orienting the magnetic field such that $h_{1}^{x}=0$, one can turn the anticrossing into a crossing, $\beta=0$. Note that also $H_{D 3}$ contains a term of the $x$ symmetry; one can still get $h_{1}^{x}=0$ since this term only slightly shifts the required position of the magnetic field. ${ }^{20}$ Changing the anticrossing into a crossing has profound consequences on the spin relaxation time, as was found in Ref. 20. As we will see below, this is similarly important also for the electrically induced spin resonance.

There are other possible formulations of the unitary transformation Eq. (12). The transformation was first used in the context of quantum dots in Ref. 37 which neglected the cubic Dresselhaus term, but kept the corrections of the third order in the spin-orbit couplings. In our notation, this correction is

$$
H_{\mathrm{lin}}^{(3)}=\left[S, H_{\mathrm{lin}}^{(2)}\right] / 3 \text {. }
$$

This term, which we neglected, together with Eq. (15), was interpreted as a vector potential of a spin-orbit originated magnetic field. ${ }^{37}$

If the confining potential is harmonic ( $d=0$ in out model), the unitary transformation can be generalized to remove explicitly also the lowest order mixed Zeeman-spin-orbit term $H_{Z}^{(2)}{ }^{38}$ The price to be paid is the appearance of spin dependent mass and other parameters. However, this possibility is specific to the potential form and nothing can be done with the cubic Dresselhaus term.

An elegant form of the unitary transformation together with the perturbation theory is worked out in Ref. 17, where an effective Hamiltonian for a set of degenerate states is derived in a compact form using an inverse of Liouville superoperator. However, the inverse is not known for other than harmonic potentials if the Zeeman term is present; the inverse is not known at all for the cubic Dresselhaus term.

The effective Hamiltonian presented here is independent of the form of the confinement potential and reveals the symmetry of the spin-dependent perturbations. In a symmetric potential, such as our double dot, simply by inspecting the symmetry of the terms allows us to identify the term responsible for a given process, be it spin relaxation or electrically induced transition. Equations (15)-(17) hold also if an out of plane component of the magnetic field $B_{z}$ is present, provided that (i) the operator $\mathbf{k}$ includes also the vector potential of this component, $\mathbf{k} \rightarrow-i \hbar \boldsymbol{\nabla}+e \mathbf{A}\left(B_{z}\right)$, and (ii) there is an additional contribution to $H_{Z}^{(2)}$, proportional to $B_{z}$ (see Ref. 28).
Using the effective spin-orbit Hamiltonian and the approximations for the eigenstates, we now quantify the individual contributions of the oscillating fields to the matrix element $\Omega_{b a}$. We will point to the origin of these contributions and show how they can be used to control the electron spin and orbital resonances.

\section{MATRIX ELEMENTS: SPIN RESONANCE}

By spin resonance, we call processes in which the two resonant states are the ground state $\bar{\Gamma}_{1 \uparrow}^{00}$ and its Zeeman split counterpart $\bar{\Gamma}_{1 \downarrow}^{00}$. We label the corresponding transition matrix element by the subscript "spin,"

$$
\Omega_{\text {spin }}^{F}=\left\langle\bar{\Gamma}_{1 \uparrow}^{00}\left|\hat{\Omega}^{F}\right| \bar{\Gamma}_{1 \downarrow}^{00}\right\rangle,
$$

where the superscript $F$ stands for the particular part of $\hat{\Omega}$ we consider.

Before we go into specifics, we recall the Van Vleck cancellation which is present in Eq. (27) due to time reversal symmetry. Consider a Hermitian operator $\hat{O}$. Let us write the matrix element in the form

$$
\left\langle\bar{\Gamma}_{1 \uparrow}^{00}|\hat{O}| \bar{\Gamma}_{1 \downarrow}^{00}\right\rangle=\left\langle\Gamma_{1 \uparrow}^{00}|\hat{O}| \Gamma_{1 \downarrow}^{00}\right\rangle+\delta O,
$$

where we singled out the contribution due to spin-orbit coupling as $\delta O$. If the first term is different from zero (the unperturbed states are coupled by $\hat{O}), \delta O$ can be usually neglected. If the first term vanishes, and we are away from an anticrossing, $\beta \ll 1$, the time reversal symmetry gives an important information about $\delta O$. Indeed, if $\hat{O}$ has a definite time reversal symmetry, $T(\hat{O})=1(-1)$ when being symmetric (antisymmetric), using Eqs. (21) and (22) for the matrix element in the lowest order in $H_{1}$, we get ${ }^{30,39}$

$$
\delta O=\sum_{i, j, \sigma}\left\langle\Gamma_{1 \uparrow}^{00} \hat{O} \Gamma_{i \sigma}^{j}\right\rangle\left\langle\Gamma_{i \sigma}^{j} H_{1} \Gamma_{1 \downarrow}^{00}\right\rangle\left(\frac{1}{E_{1 \downarrow}^{00}-E_{i \sigma}^{j}}-\frac{T\left(H_{1}\right) T(\hat{O})}{E_{1 \uparrow}^{00}-E_{i,-\sigma}^{j}}\right),
$$

where $i$ denotes the symmetry class, $j$ denotes, for brevity, both upper orbital indices, and $\sigma$ denotes the spin. In this lowest order, the contributions from the constituents of $H_{1}$ are additive and can be considered separately. Therefore, the first order contributions of the terms with the same time reversal symmetry as $\hat{O}$ [that is if $\left.T\left(H_{1}\right) T(\hat{O})=1\right]$ will be suppressed by a factor of the order of $\mu B / E_{0}$, compared to the matrix elements such as Eq. (27), but between states with different spatial indices. At zero magnetic field, the two terms in the brackets in Eq. (29) cancel exactly-this is referred to as the Van Vleck cancellation. Near the anticrossing, the terms containing coefficients $\alpha$ and $\beta$ dominate other terms in Eqs. (22) and (23) and the matrix elements are then proportional to these coefficients; the Van Vleck cancellation does not occur.

The above general results can be applied to the spin resonance due to magnetic and electric fields. The oscillating magnetic field $\left[\hbar \hat{\Omega}=\mu \mathcal{B}_{z} \sigma_{z}\right]$ couples directly the unperturbed states, 
TABLE I. Analytical approximations for the dipole matrix elements and energy differences. For each quantity the definition, unit, expression, and limits for small and large interdot distances are given. In some cases, the expression is too lengthy and only the asymptotics are given. The expression for $\delta E_{t}$ is given in Ref. 22. The interdot distance measured in the units of the confinement length is used, $\mathcal{D}=d / l_{0}$.

\begin{tabular}{lccccc}
\hline \hline & Definition & Unit & Expression & $\mathcal{D} \ll 1$ & $\mathcal{D} \gg 1$ \\
\hline $\bar{X}_{1}$ & $\left\langle\Gamma_{2}^{10}|x| \Gamma_{1}^{00}\right\rangle$ & $l_{0}$ & $\frac{\mathcal{D}}{\sqrt{1-e^{-2 \mathcal{D}^{2}}}}$ & $\frac{1}{\sqrt{2}}$ & $\mathcal{D}$ \\
$\bar{X}_{2}$ & $\left\langle\Gamma_{2}^{31}|x| \Gamma_{1}^{00}\right\rangle$ & $l_{0}$ & $-\frac{3 \mathcal{D}^{2}}{\sqrt{4}}$ & $\frac{1}{\sqrt{2}}$ \\
$\bar{Y}_{1}$ & $\left\langle\Gamma_{4}^{11}|y| \Gamma_{1}^{00}\right\rangle$ & $l_{0}$ & $\frac{1}{\sqrt{2}}$ & $\frac{1}{\sqrt{2}}$ \\
$\overline{X Y}$ & $\left\langle\Gamma_{3}^{21}\left|k_{x} k_{y}\right| \Gamma_{1}^{00}\right\rangle$ & $l_{0}^{-2}$ & $-\frac{\mathcal{D}}{\sqrt{2}} \frac{e^{-\mathcal{D}^{2}}}{\sqrt{1} \delta E_{t}^{e^{-2 \mathcal{D}^{2}}}}$ & $-\frac{1}{2}$ & $-\frac{\mathcal{D}}{\sqrt{2}} e^{-\mathcal{D}^{2}}$ \\
& $E_{2}^{10}-E_{1}^{00}$ & $E_{0}$ & & 3 & $\mathcal{D} e^{-\mathcal{D}^{2}}$ \\
& $E_{2}^{31}-E_{1}^{00}$ & $E_{0}$ & 1 & 1 & 1 \\
& $E_{4}^{11}-E_{1}^{00}$ & $E_{0}$ & & & 1 \\
\hline \hline
\end{tabular}

$$
\Omega_{\text {spin }}^{\mathcal{B}_{z}}=\alpha \mu \mathcal{B}_{z}
$$

so that we can neglect the spin-orbit contribution to the matrix element, $\delta \Omega$.

On the other hand, the electric field dipole operator $(\hbar \hat{\Omega}$ $=e \mathcal{E}$.r) does not couple the unperturbed states. As $\hat{\Omega}$ is now time reversal symmetric, the contributions of all terms in $H_{1}$ but $H_{Z}^{(2)}$ are suppressed. For the electric field along the rotated $\hat{x}$ axis, the matrix element at the anticrossing is

$$
\Omega_{\text {spin }}^{\mathcal{E}_{x}}=\beta e \mathcal{E}_{x} \bar{X}_{1}^{\dagger} .
$$

Away from the anticrossing,

$$
\Omega_{\text {spin }}^{\mathcal{E}_{x}}=-e \mathcal{E}_{x} h_{1}^{x} \mu B \sum_{j}\left|\bar{X}_{j}\right|^{2} \frac{2\left(E_{2}^{j}-E_{1}^{00}\right)}{\left(E_{2}^{j}-E_{1}^{00}\right)^{2}-4(\mu B)^{2}} .
$$

The spatial symmetry (here $x$ ) of the dipole operator selects only eigenfunctions of the symmetry $x$ in the sum. Only $H_{Z}^{(2)}$ [Eq. (16)] contains a term of the $x$ symmetry, proportional to $h_{1}^{x}$. In the above sum, each state $j$ (with energy $E_{2}^{j}$ ) contributes proportionally to its dipole matrix element $\bar{X}_{j}$. To get an analytical result close to the numerics, one needs to include the two lowest eigenfunctions in the sum in Eq. (32).

If the electric field is along the rotated $\hat{y}$ axis, the anticrossing does not influence the matrix element, since $y$ dipole operator of the electric field does not couple the ground and anticrossing states. Then, an analogous expression to Eq. (32) holds at (up to a factor $\alpha$ multiplying some terms in the sum) or away from the anticrossing,

$$
\Omega_{\text {spin }}^{\mathcal{E}_{y}}=-e \mathcal{E}_{y} h_{1}^{y} \mu B \sum_{j}\left|\bar{Y}_{j}\right|^{2} \frac{2\left(E_{4}^{j}-E_{1}^{00}\right)}{\left(E_{4}^{j}-E_{1}^{00}\right)^{2}-4(\mu B)^{2}} .
$$

Here, it is enough to include the lowest eigenfunction of $y$ symmetry in the sum. The dipole elements and the energy differences, computed by approximating the unperturbed functions $\Gamma$ by symmetrized single dot orbitals, ${ }^{22}$ are summarized in Table I.

Fully numerical results for the matrix elements as a function of the magnetic field are shown in Fig. 3(a). The matrix element of the magnetic field is constant, up to a narrow region of suppression due to $\alpha$, since it depends only on the strength of the oscillating magnetic field [Eq. (30)]. The matrix elements of the electric field [Eqs. (32) and (33)] are proportional to the Zeeman energy $\mu B$ - the spin resonance is more sensitive to electric disturbances as the magnetic field grows, while at zero magnetic field, the electric field is ineffective. At the anticrossing, $\Omega_{\text {spin }}^{\mathcal{E}_{x}}$ is strongly enhanced (by 2 orders of magnitude) as described by Eq. (31), while $\Omega_{\text {spin }}^{\mathcal{E}_{y}}$ develops a small dip similar to $\Omega_{\text {spin }}^{\mathcal{B}_{z}}$

It can be seen in Fig. 3(b), where the matrix elements are plotted as functions of the tunneling energy, that the spin resonance is much more sensitive to the electric field along the double dots $x$ axis than to a perpendicular field. This

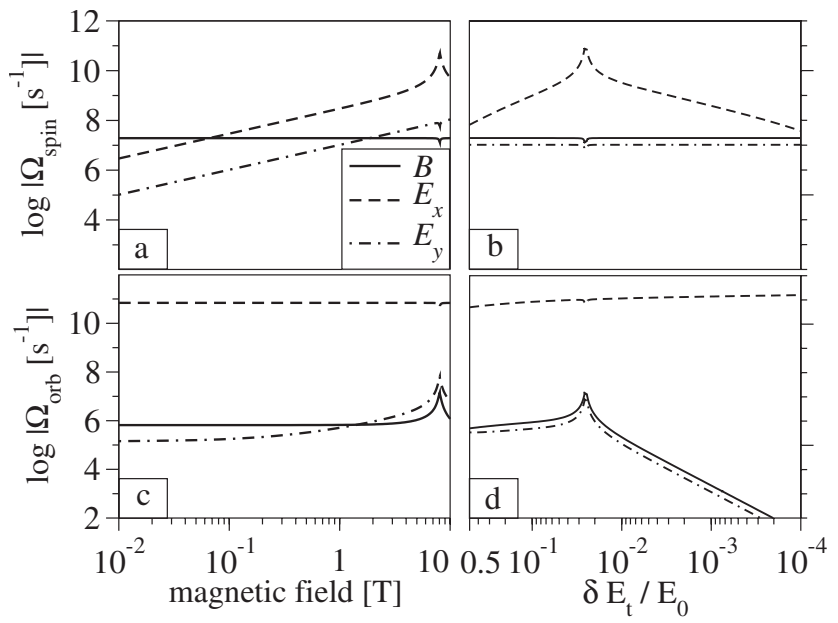

FIG. 3. Calculated matrix elements between the resonant states due to magnetic and electric oscillating fields. The two upper panels [(a) and (b)] show the matrix elements $\Omega_{\text {spin }}$ for the spin resonance, while the two lower panels show orbital resonance elements $\Omega_{\text {orb }}$. On the left, in (a) and (c), the elements are functions of the static magnetic field, with a fixed tunneling energy of $20 \%$ of the confinement energy. On the right, in (b) and (d), the elements are functions of the tunneling energy at a fixed magnetic field $B=1 \mathrm{~T}$. The dots are oriented along [100], while the static magnetic field lies along [010]. 


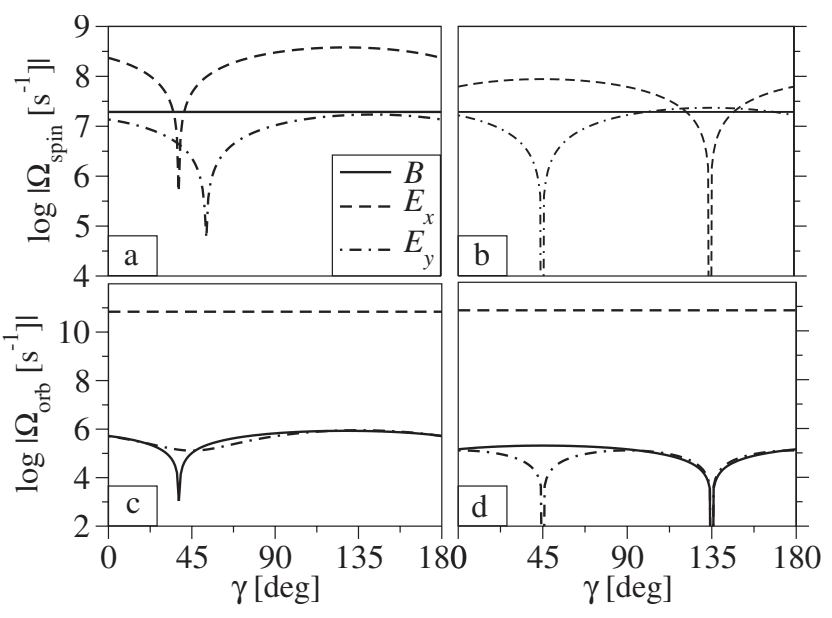

FIG. 4. Calculated matrix elements for the spin [upper two panels (a) and (b)] and the orbital [lower two panels (c) and (d)] resonance due to oscillating magnetic and electric fields as functions of $\gamma$, the orientation of the static magnetic field, $B=1 \mathrm{~T}$. The tunneling energy is $20 \%$ of the confinement energy. On the left, in (a) and (c), the dots are oriented along [100], that is, $\delta=0$. On the right, in (b) and (d), the dots are oriented along [110], $\delta=45^{\circ}$.

difference is strengthened at the anticrossing. Only in the truly single dot case $(d=0$ or $d=\infty)$, the electric field influence is isotropic. We can also conclude from the single dot values that the matrix elements of a magnetic field of $1 \mathrm{mT}$ and an electric field of $10^{3} \mathrm{~V} / \mathrm{m}$ are comparable in magnitude in the static magnetic field of the order of teslas. This means that in the experiment, ${ }^{16}$ in which no electrically induced signal was observed, the electric field is likely considerably lower than the estimated value of $10^{4} \mathrm{~V} / \mathrm{m}$.

Similar to the spin relaxation rates, ${ }^{20,40}$ the matrix element of the resonant electric field is highly anisotropic. The possible control over the resonance is demonstrated in Fig. 4(a), where the matrix elements are shown as functions of the orientation of the static magnetic field. The magnetic field matrix element is independent on $\gamma$, as follows from Eq. (30). The electric field matrix elements are anisotropic, with the dependence given by the effective spin-orbit couplings $h_{1}^{x}$ and $h_{1}^{y}$. By a proper orientation of the static magnetic field, it is thus possible to turn off the contribution due to the electric field pointed along a given direction. In particular, the electric field along $\hat{x}$ is not effective $\left(h_{1}^{x}=0\right)$ at $\gamma$ $=\arctan \left(l_{D} / l_{B R}\right) \approx 38^{\circ}$. The electric field along $\hat{y}$ is ineffective if $\gamma=\arctan \left(l_{B R} / l_{D}\right) \approx 58^{\circ}$ since here $h_{1}^{y}=0$. These conditions were obtained from Eqs. (19) and (20) by setting $\delta=0$ (the dots oriented along [100]). Different orientations of the dots change the conditions for the effective spin-orbit couplings to be zero. For example, in Fig. 4(b), the dots are oriented along [110], that is, $\delta=45^{\circ}$ and the effective couplings $h_{1}^{x}$ and $h_{1}^{y}$ are zero at $\gamma=45^{\circ}$ and $135^{\circ}$, respectively, independent of the spin-orbit parameters. If the electric field points along a general direction, it is still possible to turn the matrix element off by properly orienting the magnetic field. However, in a general case, the desired position of the magnetic field is defined not only by the effective couplings $h_{1}^{x}$ and $h_{1}^{y}$ but also by all terms in Eqs. (32) and (33).
In the easy passage configuration, defined by $h_{1}^{x}=0$, the spin relaxation time does not suffer a drastic suppression due to the anticrossing, as was shown in Ref. 20. In addition, the spin resonance is insensitive to the otherwise most effective electric field component-along $\hat{x}$. Such electric fields are inevitably present if the spin qubit is manipulated by an onchip generated magnetic field. ${ }^{16}$ On the other hand, on-chip manipulations seem inevitable in a scalable system, where it must be possible to address the qubits selectively. The easy passage configuration thus protects the spin against the unwanted electric fields and provides a stable Rabi frequency over a wide range of parameters values, if the qubit is manipulated by an oscillating magnetic field.

\section{MATRIX ELEMENTS: ORBITAL RESONANCE}

By orbital resonance, we mean processes in which the the resonant states are the lowest orbital states, so that the transition matrix element is

$$
\Omega_{\text {orb }}^{F}=\left\langle\bar{\Gamma}_{1 \uparrow}^{00}\left|\hat{\Omega}^{F}\right| \bar{\Gamma}_{2 \uparrow}^{00}\right\rangle .
$$

A similar Van Vleck suppression as in Eq. (29) takes place also now, if the operator $\hat{O}$ acts only on the spin subspace (that is, it is the Zeeman term). This suppression again favors the contribution due to $H_{Z}^{(2)}$ compared to the rest of $H_{1}$. If the anticrossing, dominates the matrix element due to $\mathcal{B}_{z}$ is $\Omega_{\mathrm{orb}}^{\mathcal{B}_{z}}=-\beta^{\dagger} \mu \mathcal{B}_{z}$, while away from the anticrossing, we get

$$
\Omega_{\text {orb }}^{\mathcal{B}_{z}}=-\mu \mathcal{B}_{z} h_{1}^{x} \bar{X}_{1} \frac{\left(E_{2}^{10}-E_{1}^{00}\right)^{2}}{\left(E_{2}^{10}-E_{1}^{00}\right)^{2}-4(\mu B)^{2}} .
$$

Contrary to the case of electrically induced spin resonance, the oscillating magnetic field can induce transitions also at zero static magnetic field, as seen in Fig. 3(c). However, the matrix element of the magnetic field becomes unimportant if the electric field in the $x$ direction is present since such an electric field is much more efficient for the orbital resonance,

$$
\Omega_{\text {orb }}^{\mathcal{E}_{x}}=e \mathcal{E}_{x} \bar{X}_{1},
$$

because it couples unperturbed states directly.

If the electric field is oriented along $\hat{y}$, it is much less effective because the linear spin-orbit terms do not contribute in the first order. Here, for a nonzero matrix element in Eq. (34), the perturbation $H_{1}$ has to contain a term which is spin diagonal with the spatial symmetry $x y$. The only such a term in $H_{1}$ is the term originating in the first term of $H_{D 3}^{(2)}$ [Eq. (17)]. After the rotation of the coordinate system, this term becomes $-\left(2 \gamma_{c} / l_{B R}\right) \cos (2 \delta) k_{x} k_{y}$, leading to the matrix element,

$$
\Omega_{\mathrm{orb}}^{\mathcal{E}_{y}}=e \mathcal{E}_{y} \bar{Y}_{1} \frac{\gamma_{c}}{l_{B R}} \cos 2 \delta \overline{X Y} \frac{4\left(E_{1}^{00}-E_{2}^{10}\right)}{\left(E_{4}^{11}-E_{1}^{00}\right)^{2}-\left(E_{2}^{10}-E_{1}^{00}\right)^{2}} .
$$

In small magnetic fields $(\$ 1 \mathrm{~T})$, this contribution dominates the matrix element compared to the contributions from other parts in $H_{1}$, such as $H_{Z}^{(2)}$, contributing in the second order. Note that there is no term with appropriate symmetry (spin 
diagonal, spatially $x y$ ) of $H_{1}$ coming from a mixture of $H_{D}$ and $H_{D 3}$, making $\Omega_{\text {orb }}^{\mathcal{E}_{y}}$ a specific effect due to the mixed cubic Dresselhaus and Bychkov-Rashba interactions. This example demonstrates the usefulness of the information about the symmetry contained in Eqs. (15)-(17). By simple inspection of the symmetry, one can recognize which term needs to be considered for a specific situation.

The dependence of the matrix elements on the static magnetic field orientation $\gamma$ is shown in Figs. 4(c) and 4(d). The magnetic field matrix element is proportional to $h_{1}^{x}$ [see Eq. (35)]. The direct coupling through the electric field along $\hat{x}$ is independent of $\gamma$. The matrix element of the electric field along $\hat{y}$, as given in Eq. (37), is independent of $\gamma$ and cannot be set to zero by changing the magnetic field orientation, as seen in Fig. 4(c). However, there is some dependence to be seen and the dependence is striking for a orientation of the dots. The reason is that Eq. (37) is the dominant contribution to the matrix element only up to a certain value of the static magnetic field-in higher fields, the second order contribution from $H_{Z}^{(2)}$ will dominate. Since there is already a visible dependence in Fig. 4(c), we can estimate the crossover magnetic field to be $1 \mathrm{~T}$ for our parameters. In Fig. 4(d), the contribution of Eq. (37) is zero exactly since $\delta=45^{\circ}$. Therefore, the second order contribution to the matrix element coming from $H_{Z}^{(2)}$ is seen. The possible dependence of the matrix element on $\gamma$ can decide whether the matrix element is induced by linear spin-orbit terms (depends on $\gamma$ ) or the mixed cubic-linear terms (does not depend on $\gamma$ ). This could be used as a detection for the presence of the cubic Dresselhaus term. Unless the electric field is oriented exactly along the $y$ axis, no oscillating magnetic field influence or anisotropy can be observed due to the high effectiveness of the electric field along $\hat{x}$.

After having analyzed the control over the field matrix element or, to the same effect, over the Rabi frequency, we will now study the steady state solution of the density matrix. We will show that the Rabi frequency and decoherence, which have been obtained in Refs. 10 and 16 from the observation of the decaying Rabi oscillations, can be obtained alternatively from the steady state current measurement.

\section{RESONANT FIELD INFLUENCE IN THE STEADY STATE}

In this section, we are interested in the steady state solution of the density matrix, denoted by $\bar{\rho}$ and defined as the solution with constant occupations,

$$
\left(\partial_{t}^{\mathrm{ph}}+\partial_{t}^{\mathrm{of}}\right) \bar{\rho}_{i i}=0, \quad \forall i,
$$

where the two contributions to the time derivative are those in Eqs. (7a), (7b), (9a), and (9b). Even though it is not currently measurable in a single electron system, we include in our list of interesting steady state parameters the absorption,

$$
W=\partial_{t}^{\mathrm{of}} \sum_{i} E_{i} \bar{\rho}_{i i},
$$

defined as the energy gain of the electron due to the oscillating field.
After the decay of the Rabi oscillations, the system is in the steady state, where the occupations are constant. In this case, the time derivative of the density matrix due to the oscillating field [Eqs. (9a) and (9b)] can be simplified to (see Ref. 3 for the derivation)

$$
\partial_{t}^{\text {of }} \rho_{a a}=-\partial_{t}^{\text {of }} \rho_{b b}=2\left(\rho_{b b}-\rho_{a a}\right) J,
$$

where the induced rate

$$
J=\frac{\left|\Omega_{b a}\right|^{2}}{4} \frac{\gamma_{b a}}{\Delta^{2}+\gamma_{b a}^{2}} .
$$

The zero time derivative of the occupations in the steady state can be interpreted as a balance between two competing processes: relaxation [Eqs. (7a) and (7b)] which drives the system toward the thermodynamical equilibrium $\left(\rho_{b b} / \rho_{a a}\right.$ $\left.=\Gamma_{a b} / \Gamma_{b a}\right)$, and an oscillating field induced transition [Eq. (40)] equilibrating occupations of the resonant states $\left(\rho_{b b}\right.$ $\left.=\rho_{a a}\right)$. The effectiveness of the oscillating field in driving the system out of thermal equilibrium is characterized by the induced rate $J$ [Eq. (41)]. Away from the resonance, the oscillating field is less effective in influencing the system, which is reflected by the (Lorentzian shape) decay of the induced rate.

Our numerical strategy to obtain the steady state density matrix $\bar{\rho}$ is as follows: We diagonalize the coupled dots electron Hamiltonian [Eq. (1)] (Ref. 22) and compute the relaxation rates using Fermi golden rule. ${ }^{28}$ We choose a pair of resonant states, $\{\mathrm{a}, \mathrm{b}\}$, and after evaluating $\Omega_{b a}$, we find the induced rate according to Eq. (41). Finally, we find the steady state density matrix by solving the set of linear equations defined by Eq. (38). A different method, with the oscillating field treated exactly, was used for single dot in intense oscillating fields, ${ }^{41,42} 3$ orders of magnitude larger than the fields considered here.

We can analytically reproduce the numerical results by the two state approximation discussed in the above. The physics is then characterized by the number

$$
J_{0}^{r}=\left.\Gamma_{b a}^{-1} J\right|_{\omega=\omega_{b a}}=\left|\Omega_{b a}\right|^{2} \frac{1}{4 \gamma_{b a} \Gamma_{b a}},
$$

which is the induced rate at the resonance, measured in the units of the relaxation rate between the resonant states.

Two limits can be identified, according to $J_{0}^{r}$. If the induced rate dominates the relaxation, $J_{0}^{r} \gg 1$, the occupations of the two resonant states are close to being equal, while if $J_{0}^{r} \ll 1$, the system is close to the thermal equilibrium. The interpretation of $2 J$ as the electron outscattering rate due to the oscillating field, as it follows from Eq. (40), is reassured by the result form the absorption. We expect the absorption to be proportional to a transition rate from the excited state to the ground state times the energy dissipated at this transition. If $J_{0}^{r} \ll 1$, the transition rate is $2 J$. In the opposite limit, $J_{0}^{r}$ $\gg 1$, the outscattering due to the oscillating field is strong and the transition rate for the dissipation is limited by the relaxation rate. The frequency full widths at half maximum (FWHMs) also differ for the two limits (see Table II for analytical results). 
TABLE II. (a) Steady state, value at resonance, and frequency full width at half maximum (FWHM) $\delta \omega_{1 / 2}$ squared for the excited state occupation $\bar{\rho}_{b b}$, the induced rate $J$, and absorption $W$. Note that the FWHM of the excited population is defined only if the temperature is low enough such that $J_{0}^{r} \geqslant \tau(1+\tau) /(1-3 \tau)$. (b) The value at the resonance and frequency full width at half maximum of the excited population and absorption in the two limits.

\begin{tabular}{|c|c|c|c|}
\hline (a) & Steady state & At resonance & $\operatorname{FWHM}\left(\delta \omega_{1 / 2}^{2}\right)$ \\
\hline \multirow[t]{2}{*}{$\bar{\rho}_{b b}$} & $J+\tau \Gamma_{b a}$ & $J_{0}^{r}+\tau$ & $8 J_{0}^{r}\left(1+J_{0}^{r}\right)+4 \tau\left(1+\tau+3 J_{0}^{r}\right)$ \\
\hline & $\overline{2 J+\Gamma_{b a}(1+\tau)}$ & $\overline{2 J_{0}^{r}+1+\tau}$ & $J_{0}^{r}-\tau\left(1+\tau+3 J_{0}^{r}\right)$ \\
\hline$J$ & $\underline{\left|\Omega_{b a}\right|^{2} \gamma_{b a}}$ & $\left|\Omega_{b a}\right|^{2} / 4 \gamma_{b a}$ & $4 \gamma_{b a}^{2}$ \\
\hline$W$ & $\overline{4 \Delta^{2}+\left(1 \gamma_{b \boldsymbol{t}}^{2}\right)}$ & $2(1-\tau)$ & $4\left(1+\tau+2 J_{0}^{r}\right)$ \\
\hline (b) & $\begin{array}{c}L_{b a}{ }^{L} \\
\text { Limit } \\
\end{array}$ & $\begin{array}{l}L_{b a}{ }^{J} \\
\text { At resonance }\end{array}$ & $\operatorname{FWHM}^{1+\tau}\left(\delta \omega_{1 / 2}^{2}\right)$ \\
\hline $\bar{\rho}_{b b}$ & $J_{0}^{r} \gg 1$ & $1 / 2-(1-\tau) / 2 J_{0}^{r}$ & $2\left|\Omega_{b a}\right|^{2} \gamma_{b a} / \Gamma_{b a}(1-3 \tau)$ \\
\hline $\bar{\rho}_{b b}$ & $J_{0}^{r} \ll 1$ & $\frac{\tau}{1-\tau}+J_{0}^{r}(1-\tau) /(1+\tau)^{2}$ & $4 \gamma_{b a}^{2}$ \\
\hline$W$ & $J_{0}^{r} \gg 1$ & ${ }^{1+\tau_{E_{b a}} \Gamma_{b a}(1-\tau)}$ & $2\left|\Omega_{b a}\right|^{2} \gamma_{b a} / \Gamma_{b a}(1+\tau)$ \\
\hline$W$ & $J_{0}^{r} \ll 1$ & $2 E_{b a} J^{r}(1-\tau) /(1+\tau)$ & $4 \gamma_{b a}^{2}$ \\
\hline
\end{tabular}

Figure 5 presents our numerical results for the induced rate, excited population width, and decoherence as functions of the tunneling energy for the spin and orbital resonance. Both resonances are in the regime of $J_{0}^{r} \gg 1$, where the decoherence is revealed by the FWHM of the induced rate (see Table II), while the relaxation rate can be obtained if both the induced rate at resonance and FWHM of the excited population are known, too. Due to Eq. (10), the relaxation rate is indiscernible from the decoherence in the figure and $J_{0}^{r}$ can be directly determined. For the spin resonance, $J_{0}^{r}$ varies between $10^{5}$ and $10^{11}$; the limit expressions in Table II are exact with this precision. The upward dips in FWHM and the decoherence rate are due to the anticrossing of the spin and

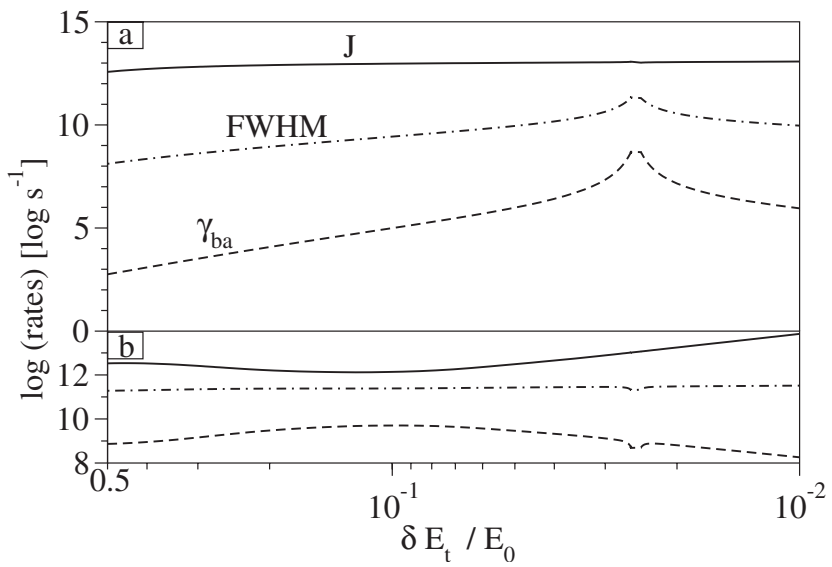

FIG. 5. Calculated induced rate $J$ at resonance (solid line), decoherence $\gamma_{b a}$ (dashed line), and the FWHM of the excited population (dot-dashed line) as functions of the ratio of the tunneling energy $\delta E_{t}$ and the confinement energy $E_{0}$ for (a) spin resonance and (b) orbital resonance. The static in-plane magnetic field is $B$ $=1 \mathrm{~T}$. If the solid line is above (under) the dashed one, it means that $J_{0}^{r}>1\left(J_{0}^{r}<1\right)$. The dots are oriented along [100], while the static magnetic field lies along [010]. orbital states. ${ }^{22}$ It is interesting that the induced rate is not influenced by the anticrossing. This is because both the square of the matrix element and the decoherence (equal to the relaxation) in Eq. (41) depend on the anticrossing in the same way and the contributions cancel. Also, note that the rates characterizing the oscillating field are very different in the transient and the steady state regimes. While the steady state characteristic rate $J$ is $\sim 10^{13} \mathrm{~s}^{-1}$, looking at Fig. 3(b), one can see that the Rabi frequency for the same parameters is only $\sim 10^{8} \mathrm{~s}^{-1}$.

Compared to the spin resonance, the orbital resonance is much less sensitive to the anticrossing since only in a very narrow region at the anticrossing the relaxation rate acquires a factor of one-half. ${ }^{28}$ One also sees that $J_{0}^{r}$ is smaller, meaning we are closer to the regime of $J_{0}^{r}<1$ which can be reached by lowering the amplitude of the oscillating electric field. In that regime, the decoherence can be obtained from the FWHM of the excited population or from the induced rate.

After identifying the appropriate regime of high or low induced rate, one can obtain the decoherence rate and the Rabi frequency using the expressions from Table II provided the induced rate $J$ and the excited state population $\bar{\rho}_{b b}$ (and their full widths) can be measured. In turn, these two parameters could be measured if the dot is connected to leads and a current flows through the dot, as shown theoretically in Ref. 43. In Ref. 3, it is shown, using a simpler model, that the measurement can be done by changing the coupling between the dot and the leads. Namely, for a small coupling, the current is proportional to the excited state population, while for a large coupling, the current measures the induced rate.

Even though probing of electron decoherence using the steady state quantities, as we discussed it here, was proposed relatively long time ago, up to now it was not experimentally achieved. Among problems hindering such observation is heating induced by the microwave field, which enables the electron in the ground state to escape the dot by a thermal 
excitation. Similarly, the electron can be expelled from the dot by the oscillating electric field in a process known as the photon assisted tunneling (see Ref. 4).

\section{CONCLUSIONS}

We have studied electrically and magnetically induced spin and orbital resonances of a single electron confined in coupled lateral quantum dots. We have taken into account relaxation and decoherence due to an acoustic phonon environment, with the rates computed by Fermi golden rule. Resonant oscillating electromagnetic fields are capable to induce transitions between electron eigenstates. We have focused on the oscillating field matrix elements, equal to the Rabi frequency, for the spin and orbital resonances.

We have given an effective spin-orbit Hamiltonian which allows us to quantify the spin-orbit influence on the transition matrix element using symmetry considerations. Specifically, for electrically induced spin resonance, we have shown how the spin-orbit anisotropy allows us to control the matrix element by both the strength and the orientation of the static magnetic field. These conclusions give hints for optimal quantum dot configurations for the case when (i) the spin is manipulated by an oscillating electric field, whereas its influence is desired to be maximized, and (ii) the spin manipulated by an oscillating magnetic field, when the effect of the electric field on the spin is desired to be minimized. Connecting with our previous work, we have found that the easy passage provides not only increase spin relaxation times, but also stability against electric field disturbances, making it a suitable arrangement for spin qubit realization.

In a double dot, the electric field is most effective in spin manipulation if it lies along the dots' axis and the matrix element is strongly influenced by the anticrossing (a crossing of different spin states lifted by spin-orbit interactions). An important feature is that the electric field is less effective if the magnitude of the static magnetic field is lowered. Oscillating electric fields of order of $1000 \mathrm{~V} / \mathrm{m}$ can be more effective than oscillating magnetic fields of $1 \mathrm{mT}$ if the static magnetic field is of the order of a tesla. For these parameters in a GaAs quantum dot, the Rabi frequency of $1 \mathrm{GHz}$ is achievable for the spin manipulation using an electric field.

Finally, we have studied the influence of the resonant fields on the steady state. We proposed the induced rate as a single characteristic parameter. We have analyzed the steady state occupations, the induced rate, and the absorption, as well as the full widths for both spin and orbital resonances. We have used those results to show how to obtain the decoherence rate and the Rabi frequency from the steady state characteristics. In turn, these characteristics can be obtained from a steady state current measurement.

\section{ACKNOWLEDGMENTS}

We would like to thank Lieven M. K. Vandersypen, Frank H. L. Koppens, and Eugene Ya. Sherman for useful discussions. This work was supported by the U.S. ONR and one of us (P.S.) was supported by project RPEU-0014-06.
${ }^{1}$ D. Loss and D. P. DiVincenzo, Phys. Rev. A 57, 120 (1998).

${ }^{2}$ V. Cerletti, W. A. Coish, O. Gywat, and D. Loss, Nanotechnology 16, R27 (2005).

${ }^{3}$ J. Fabian, A. Matos-Abiague, C. Ertler, P. Stano, and I. Žutić, Acta Phys. Slov. 57, 565 (2007).

${ }^{4}$ R. Hanson, L. P. Kouwenhoven, J. R. Petta, S. Tarucha, and L. M. K. Vandersypen, Rev. Mod. Phys. 79, 1217 (2007).

${ }^{5}$ Y. G. Semenov and K. W. Kim, Phys. Rev. B 75, 195342 (2007).

${ }^{6}$ J. M. Elzerman, R. Hanson, L. H. Willems van Beveren, B. Witkamp, L. M. K. Vandersypen, and L. P. Kouwenhoven, Nature (London) 430, 431 (2004).

${ }^{7}$ S. Amasha, K. MacLean, I. Radu, D. M. Zumbuhl, M. A. Kastner, M. P. Hanson, and A. C. Gossard, arXiv:cond-mat/0607110 (2006).

${ }^{8}$ T. Meunier, I. T. Vink, L. H. Willems van Beveren, K.-J. Tielrooij, R. Hanson, F. H. L. Koppens, H. P. Tranitz, W. Wegscheider, L. P. Kouwenhoven, and L. M. K. Vandersypen, Phys. Rev. Lett. 98, 126601 (2007).

${ }^{9}$ T. Fujisawa, D. G. Austing, Y. Tokura, Y. Hirayama, and S. Tarucha, Nature (London) 419, 278 (2002).

${ }^{10}$ J. R. Petta, A. C. Johnson, J. M. Taylor, E. A. Laird, A. Yacoby, M. D. Lukin, C. M. Marcus, M. P. Hanson, and A. C. Gossard, Science 309, 2180 (2005).

${ }^{11}$ E. A. Laird, J. R. Petta, A. C. Johnson, C. M. Marcus, A. Yacoby, M. P. Hanson, and A. C. Gossard, Phys. Rev. Lett. 97, 056801 (2006).
${ }^{12}$ J. M. Taylor, J. R. Petta, A. C. Johnson, A. Yacoby, C. M. Marcus, and M. D. Lukin, Phys. Rev. B 76, 035315 (2007).

${ }^{13}$ D. P. DiVincenzo, Fortschr. Phys. 48, 771 (2000).

${ }^{14}$ H.-A. Engel, V. N. Golovach, D. Loss, L. M. K. Vandersypen, J. M. Elzerman, R. Hanson, and L. P. Kouwenhoven, Phys. Rev. Lett. 93, 106804 (2004).

${ }^{15}$ R. Hanson, L. H. Willems van Beveren, I. T. Vink, J. M. Elzerman, W. J. M. Naber, F. H. L. Koppens, L. P. Kouwenhoven, and L. M. K. Vandersypen, Phys. Rev. Lett. 94, 196802 (2005).

${ }^{16}$ F. H. L. Koppens, C. Buizert, K. J. Tielrooij, I. T. Vink, K. C. Nowack, T. Meunier, L. P. Kouwenhoven, and L. M. K. Vandersypen, Nature (London) 442, 766 (2006).

${ }^{17}$ V. N. Golovach, M. Borhani, and D. Loss, Phys. Rev. B 74, 165319 (2006)

${ }^{18}$ E. I. Rashba and A. L. Efros, Phys. Rev. Lett. 91, 126405 (2003).

${ }^{19}$ L. S. Levitov and E. I. Rashba, Phys. Rev. B 67, 115324 (2003).

${ }^{20}$ P. Stano and J. Fabian, Phys. Rev. Lett. 96, 186602 (2006).

${ }^{21}$ A. Pfund, I. Shorubalko, R. Leturcq, and K. Ensslin, Appl. Phys. Lett. 89, 252106 (2006).

${ }^{22}$ P. Stano and J. Fabian, Phys. Rev. B 72, 155410 (2005).

${ }^{23}$ I. Žutić, J. Fabian, and S. Das Sarma, Rev. Mod. Phys. 76, 323 (2004).

${ }^{24}$ E. I. Rashba, Fiz. Tverd. Tela (Leningrad) 2, 1224 (1960).

${ }^{25}$ Y. A. Bychkov and E. I. Rashba, J. Phys. C 17, 6039 (1984).

${ }^{26}$ G. Dresselhaus, Phys. Rev. 100, 580 (1955).

${ }^{27}$ M. Dyakonov and V. Kachorovskii, Fiz. Tekh. Poluprovodn. (S.- 
Peterburg) 20, 178 (1986).

${ }^{28}$ P. Stano and J. Fabian, Phys. Rev. B 74, 045320 (2006).

${ }^{29}$ Y. Y. Wang and M. W. Wu, Phys. Rev. B 74, 165312 (2006).

${ }^{30}$ A. V. Khaetskii and Y. V. Nazarov, Phys. Rev. B 64, 125316 (2001)

${ }^{31}$ J. I. Climente, A. Bertoni, G. Goldoni, and E. Molinari, Phys. Rev. B 75, 245330 (2007).

${ }^{32}$ K. Blum, Density Matrix Theory and Applications (Plenum, New York, 1996).

${ }^{33} \mathrm{~F}$. Koppens (private communication).

${ }^{34}$ F. Brito, H. Westfahl, Jr., A. O. Caldeira, and G. MedeirosRibeiro, arXiv:cond-mat/0703009 (2007).

${ }^{35}$ W. Nagourney, J. Sandberg, and H. Dehmelt, Phys. Rev. Lett. 56,
2797 (1986).

${ }^{36}$ F. Jelezko, T. Gaebel, I. Popa, A. Gruber, and J. Wrachtrup, Phys. Rev. Lett. 92, 076401 (2004).

${ }^{37}$ I. L. Aleiner and V. I. Falko, Phys. Rev. Lett. 87, 256801 (2001).

${ }^{38}$ M. Valín-Rodríguez, A. Puente, and L. Serra, Phys. Rev. B 69, 153308 (2004).

${ }^{39}$ J. H. Van Vleck, Phys. Rev. 57, 426 (1940).

${ }^{40}$ O. Olendski and T. V. Shahbazyan, Phys. Rev. B 75, 041306(R) (2007).

${ }^{41}$ J. H. Jiang, M. Q. Weng, and M. W. Wu, J. Appl. Phys. 100, 063709 (2006).

${ }^{42}$ J. H. Jiang and M. W. Wu, Phys. Rev. B 75, 035307 (2007).

${ }^{43}$ H.-A. Engel and D. Loss, Phys. Rev. Lett. 86, 4648 (2001). 